\title{
Un panorama sobre la cobertura educativa a nivel superior en México para personas con discapacidad
}

\author{
A panorama of the coverage at the higher educational level for people with disabilities in Mexico \\ Jonathan Alejandro González García \\ Alicia Zúñiga Llamas \\ Pedro Octavio Arce Casas
}

\begin{abstract}
RESUMEN
La mayor parte de las investigaciones sobre inclusión educativa en México se centran en los principios axiológicos que definen cómo debe ser la educación, en lugar de considerar cómo es en realidad. Han analizado escasamente el impacto de las acciones emprendidas en este rubro, así como sus alcances y limitaciones. El objetivo de esta investigación fue analizar el nivel de inclusión educativa para personas con discapacidad en el nivel superior en México. El enfoque de la investigación fue mixto, con un acercamiento exploratorio. Para esta tarea se desarrolló un indicador de desempeño, cuyo objetivo fue medir la cobertura educativa para personas con discapacidad en educación superior. Mediante el indicador se llevó a cabo un diagnóstico general de la inclusión en las diversas entidades de la República, lo que permitió conocer las brechas educativas que existen entre estas. Además se analizaron los programas gubernamentales que han buscado reducir estas desigualdades educativas. Este artículo contribuye a la agenda de la inclusión para personas con discapacidad, da cuenta de las directrices sobre cuáles aspectos habrá que mejorar, de cuáles instituciones nacionales podemos aprender y cómo el uso de herramientas tecnológicas podría contribuir a fortalecer los servicios educativos que se brindan a esta población.
\end{abstract}

Palabras clave: inclusión educativa, indicadores, educación superior, políticas públicas.

\begin{abstract}
The research about educational inclusion in Mexico has over poised its "axiological" character to the "facts" issues; that is to say, they have oriented their reflection on how education should be on equal opportunities, instead of what its current situation is. The impact of the actions that have been undertaken, as well as their scope and limitations have been scarcely analyzed. This document analyzes the educational inclusion for people with disabilities at the higher level in Mexico. The focus of the research was mixed, with an exploratory approach. For this task, a performance indicator was developed, whose objective was to measure educational coverage for people with disabilities at this school level. Through the indicator a general diagnosis of inclusion in the various entities of the Republic is offered and at the same time it allows to know the educational gaps. The government programs that have sought to reduce these educational inequalities are also analyzed. In addition, this text aims to contribute to the inclusion agenda for people with disabilities, reports the guidelines on which aspects should be improved, from which national institutions can we learn and how technological tools could contribute to strengthening educational services for this population.
\end{abstract}

Keywords: educational inclusion, indicators, higher education, public policies. 


\section{INTRODUCCIÓN}

Las investigaciones sobre inclusión educativa en México se han enfocado más en el “deber ser" que en el "ser", ha preponderado principalmente su carácter "axiológico" y en menor medida las cuestiones de "hechos", es decir, han orientado su reflexión en torno a cómo debería ser la educación en igualdad de oportunidades, y en menor prioridad a cuál es su situación actual. Hay pocos estudios sobre el impacto de las acciones que se han emprendido respecto a la inclusión educativa.

La noción inclusión educativa surgió como un conjunto de valores y principios cuyo propósito era lograr que la educación brindara acceso para todos los sectores de la población sin ninguna restricción. En ese momento, el carácter axiológico de estos principios hizo posible su reconocimiento dentro del marco legislativo en México, y permitió, además, una trascendencia de impacto social, cultural, educativa y gubernamental; sin embargo, la necesidad actual de brindar educación con igualdad de oportunidades a los sectores vulnerables de la población es reconocida, y ahora, la tarea es entender cómo lograrlo.

La inclusión educativa comprende la movilización de los diversos actores involucrados y el entramado de sus relaciones: los gobiernos, las instituciones educativas, la sociedad, las políticas públicas y la población de inclusión. Esta última está integrada por personas en situación vulnerable o de pobreza, pertenecientes a alguna etnia o con alguna discapacidad.

Debido a que cada sector de la población presenta necesidades distintas, al considerar cómo atender estas dificultades, desde el ámbito educativo, es necesario

Jonathan Alejandro González García. Profesor de tiempo completo en el Sistema de Universidad Virtual de la Universidad de Guadalajara, México. Tiene reconocimiento al perfil Prodep. Miembro del Cuerpo Académico de Investigación 862 "Gestión Estudiantil Universitaria”, adscrito al Instituto de Gestión del Conocimiento en Ambientes Virtuales del SUV. Entre sus publicaciones más reciente se encuentran "La deserción en la modalidad virtual: una mirada desde la perspectiva de los desertores" (2020) y "Trayectorias laborales esporádicas o nulas en egresados de licenciatura en el Sistema de Universidad Virtual de la Universidad de Guadalajara” (2019). Correo electrónico: jonathan.glez@udgvirtual.udg.mx. ID: https://orcid.org/0000-0002-0497-3243.

Alicia Zúñiga Llamas. Profesora de tiempo completo en el Sistema de Universidad Virtual de la Universidad de Guadalajara, México. Tiene reconocimiento al perfil Prodep. Miembro del Cuerpo Académico de Investigación 862 "Gestión Estudiantil Universitaria", adscrita al Instituto de Gestión del Conocimiento en Ambientes Virtuales del SUV. Entre sus publicaciones más reciente se encuentran "La deserción en la modalidad virtual: una mirada desde la perspectiva de los desertores" (2020) y "Trayectorias laborales esporádicas o nulas en egresados de licenciatura en el Sistema de Universidad Virtual de la Universidad de Guadalajara” (2019). Correo electrónico: alicia.zuniga@udgvirtual.udg.mx. ID: https://orcid.org/0000-0003-3679-188X.

Pedro Octavio Arce Casas. Jefe de la Unidad de Presupuestos de la Coordinación de Planeación del Sistema de Universidad Virtual, Universidad de Guadalajara, México. Es doctor en Gestión de la Educación Superior por el Centro Universitario de Ciencias Económico Administrativas de la Universidad de Guadalajara. Sus temas de investigación son Conciliación escuela, trabajos y modos de vida de estudiantes de educación superior, Representaciones de género en estudiantes de educación secundaria y Violencia de género en educación superior. Correo electrónico: pedro.arce@udgvirtual.udg.mx. ID: https://orcid. org/0000-0002-4398-6725. 
contemplar múltiples perspectivas de análisis. El objetivo de este artículo es estudiar de qué maneras pueden responderse las necesidades de las personas con discapacidad, desde el área de educación superior, en México.

Las personas con discapacidad, como población heterogénea con múltiples particularidades, tienen derecho a una educación sin distinción. Esto implica que el Estado debe respetar, proteger y garantizar que los ciudadanos en esta situación puedan ejercer este derecho, y otros, sin ningún tipo de limitante o discriminación.

El acceso a la educación superior es uno de los mecanismos de disolución de las clases sociales, de mejora en los ingresos salariales y, por ende, de la calidad de vida de los ciudadanos. En este sentido, se vuelve relevante entender el estado actual de la inclusión educativa en el nivel superior, ya que esta es el reflejo de la consecución de una trayectoria escolar satisfactoria, desde la educación básica hasta la universitaria. Estudiar el flujo y el tránsito de personas con alguna discapacidad por las instituciones de educación superior (IES) de un país brinda un panorama sobre el nivel de éxito de educación incluyente que posee la nación, ya que la educación universitaria es uno de los eslabones que componen un itinerario escolar amplio.

Por lo anterior, en este documento se crea un diagnóstico sobre la situación actual del nivel de inclusión educativa en el nivel superior en México. Para lograr esto fue desarrollado un indicador de desempeño, el cual fue utilizado para medir la cobertura de inclusión educativa en las diferentes entidades del país. Con los datos recabados fue posible conocer las brechas educativas que existen entre los estados mexicanos, y a partir de esto fue posible el análisis de algunos de los programas gubernamentales que promueven la inclusión en la educación.

\section{Antecedentes}

Para ofrecer un panorama pertinente sobre los precedentes de la inclusión educativa en México es indispensable mencionar el marco normativo, la literatura y las investigaciones que se han hecho al respecto, así como las políticas públicas que han tratado de reducir las brechas de la exclusión, pues estas últimas dan cuenta de los instrumentos, las reglas y los recursos que son destinados a la inclusión.

El marco normativo de la inclusión en la educación superior comprende un antecedente y dos convenciones a nivel internacional. El antecedente es la Declaración de Salamanca y Marco de Acción para las Necesidades Educativas Especiales (UNESCO, 1994), centrada en el reconocimiento de la igualdad de oportunidades para las personas con alguna discapacidad en centros educativos que integren a personas con y sin discapacidad. La primera convención, enunciada en la Organización de las Naciones Unidas para la Educación, la Ciencia y la Cultura (UNESCO), es la Convención sobre los Derechos de las Personas con Discapacidad (UNESCO, 2008), enfocada en los aspectos legislativos para asegurar el derecho al acceso a la educación, 
en todos sus niveles, por parte de los Estados miembros de esta organización. La segunda se llevó a cabo en Latinoamérica, fue denominada Convención Interamericana sobre la Eliminación de todas las Formas de Discriminación contra las Personas con Discapacidad (Secretaría General de la OEA, 2001), la cual busca eliminar todas las formas de discriminación contra las personas con alguna discapacidad, esto incluye el ámbito de la educación.

A nivel nacional, en México se encuentra el marco de política en el Plan Nacional de Desarrollo y el marco normativo federal respecto a la inclusión. En el primero se busca lograr el bienestar de las personas con discapacidad mediante el desarrollo integral a lo largo de su vida, así como el cambio de la cultura excluyente y discriminatoria a través de la educación, la ciencia y la tecnología.

En el ámbito legislativo, se encuentran la Ley General para las Personas con Discapacidad (Congreso de la Unión de los Estados Unidos Mexicanos, 2011), cuya última reforma es del 7 de julio del 2018, la cual tiene la intención de integrar a las personas con discapacidad mediante la creación de programas, el fortalecimiento de los existentes y el otorgamiento de becas para personas con discapacidad, y la Ley Federal para Prevenir y Eliminar la Discriminación (Congreso de la Unión de los Estados Unidos Mexicanos, 2003), en cuya última reforma, del 21 de junio del 2018, se considera que impedir el acceso a la educación y no garantizar su permanencia son actos discriminatorios.

En el ámbito internacional, la Convención Interamericana para la Eliminación de todas las Formas de Discriminación contra las Personas con Discapacidad (Secretaría General de la OEA, 2001) compromete a los Estados miembros, entre ellos México, para alcanzar sus objetivos, a implementar medidas que eliminarán la discriminación y lograrán progresivamente la integración de las personas con discapacidad en el suministro de bienes, servicios, instalaciones, programas y actividades como la educación; observables en edificios y vehículos que faciliten el acceso y que eliminen obstáculos para las personas con alguna discapacidad.

Respecto a la literatura y las investigaciones sobre el tema, se encontró que estas resaltan, en su mayoría, el sentido axiológico del problema; además, en el aspecto técnico, se encontraron trabajos basados en los marcos normativos de las instituciones y su percepción, o estudios de caso sobre experiencias de personas con algún tipo de discapacidad; por último, en menor cantidad, existen artículos de investigación que hablan sobre la tasa de ingreso a la educación superior de personas con alguna discapacidad, con el dato presentado de manera aislada y descriptiva.

En cuanto al aspecto normativo y axiológico de los estudios, las investigaciones de Cruz (2010), Cruz (2016), Pérez-Castro (2016), Vadillo y Casillas (2017) analizaron los marcos normativos referentes a la inclusión a nivel superior y la percepción que la comunidad universitaria tiene al respecto. En relación con el análisis de las expe- 
riencias que han tenido los estudiantes con alguna discapacidad se encontraron los estudios de caso de Escandón (2010), Tapia y Manoslava (2012), Villafañe, Corrales y Soto (2014).

El reporte de Toscano, Ponce, Cruz, Zapién, Contreras y Pérez (2017) muestra que la matrícula de estudiantes con alguna discapacidad en educación superior es menor a $1 \%$. Estos resultados fueron obtenidos al analizar las fuentes de información antes mencionadas y los anuarios estadísticos de la Asociación Nacional de Universidades e Instituciones de Educación Superior de México (ANUIES). ${ }^{1}$ Estos autores proponen que la inclusión en educación superior debe ser un criterio considerado por los organismos acreditadores que evalúan las instituciones. De acuerdo con lo anterior, resulta necesario tener una propuesta más completa de indicadores sobre las instituciones de educación superior mexicanas que pueda ser integrada en la agenda política.

En lo que respecta a las políticas públicas y al financiamiento como apoyo para la inclusión en México, las instituciones de educación superior (IES) están obligadas a adecuarse a las normas y políticas emanadas de los poderes fácticos, pues en este entramado de relaciones se asigna el presupuesto y los recursos extraordinarios.

Mediante la modernización de estos mecanismos gubernamentales se ha incentivado a las IES para impulsar la inclusión educativa en la nación; estas acciones se han llevado a cabo mediante programas específicos, como becas a estudiantes de grupos vulnerables y recursos extraordinarios concursables para las IES en favor de la inclusión. En estos últimos, las universidades participan con proyectos que buscan la inclusión, a fin de ser beneficiadas con recursos adicionales. Esto no quiere decir que las IES no dediquen esfuerzos a expensas de estos incentivos, algunas poseen programas de inclusión que no dependen de estos insumos. En el reporte de Vadillo y Casillas (2017) se menciona que solo 17 instituciones de educación superior en México operan programas de inclusión, las cuales se encuentran en los estados de Campeche, Chihuahua, Ciudad de México, Durango, Guanajuato, Hidalgo, Nuevo León, San Luis Potosí, Tamaulipas, Tlaxcala y Veracruz.

En el ámbito federal, una de las acciones que el gobierno destina a la inclusión educativa en el nivel superior es a través del Programa para la Inclusión y la Equidad Educativa (PIEE), creado a partir del año 2015 y aún vigente. Desde su creación, ha destinado un promedio de 70075958 de pesos anuales, lo que equivale al $0.06 \%{ }^{2}$ del presupuesto total destinado a la educación superior durante el 2018. Este recurso económico se reparte cada año entre las diversas IES públicas que concursan con proyectos de inclusión.

1 Es una asociación no gubernamental, de carácter plural, que agremia a las principales instituciones de educación superior del país, cuyo común denominador es su voluntad para promover su mejoramiento integral en los campos de la docencia, la investigación y la extensión de la cultura y los servicios (ver ANUIES, s.f.). 


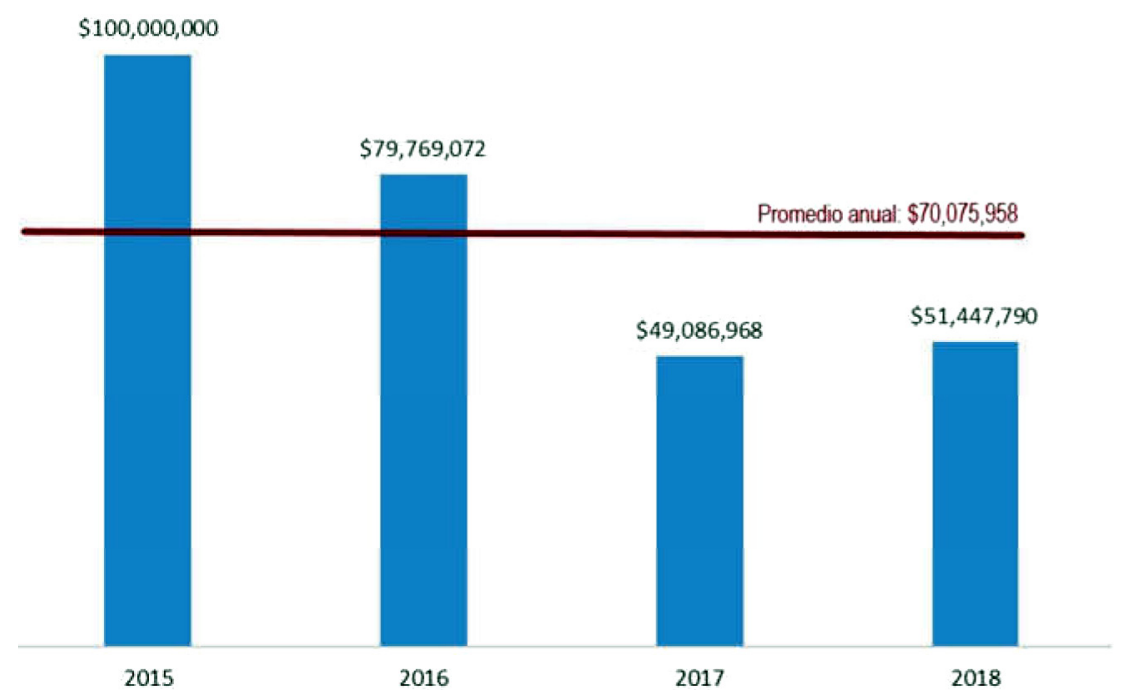

Figura 1. México: financiamiento del Programa para la Inclusión y la Equidad Educativa, 2015-2018. Fuente: Elaboración propia a partir de los datos del Programa para la Inclusión y la Equidad Educativa (Gobierno de México, s.f.).

El PIEE como estrategia política aún es incipiente, pues los recursos económicos con los que cuenta representan menos de 1\% del presupuesto total que se ejerce en educación superior pública; además este programa ha mostrado a través de los años una reducción considerable, de cerca del 50\% (ver figura 1).

Cabe destacar que la gestión de estos fondos por parte de las IES representa la mayoría de su capacidad de crecimiento en infraestructura física (construcción/obra, remodelaciones de espacios físicos y equipamiento), ya que el presupuesto anual de estas organizaciones se encuentra comprometido casi al 90\% en gasto corriente (pago de nómina y servicios). Es a través de estos fondos extraordinarios que se pueden concretar acciones específicas que consoliden la inclusión educativa; en este sentido, se vuelve indispensable el incremento de estos recursos económicos.

Otra fuente de recursos que apoya la inclusión ha sido el Programa para la Inclusión Social Prospera (creado en 1988 y aún vigente), ${ }^{3}$ el cual otorga becas a la población estudiantil de nuevo ingreso de cualquier IES pública que pertenece a sectores de población vulnerable: grupos étnicos o en condición de pobreza; sin embargo, este programa no contempla el apoyo a personas con algún tipo de discapacidad.

Existió otro programa que apoyó exclusivamente a estudiantes con discapacidad: Educación para Personas con Discapacidad (EPD) (2009-2013), cuyo objetivo fue

2 Lo que equivale a una inversión total de \$118 591800000.00 (información disponible en SHCP, s.f.).

3 Ha tenido diferentes nombres: Solidaridad, Progresa y Oportunidades (ver Gobierno de México, s.f.). 
apoyar a estudiantes con discapacidad auditiva, motriz, visual, de lenguaje o intelectual para que pudieran continuar o concluir sus estudios de educación media superior y superior. En la educación universitaria, el apoyo se gestionaba a través del Programa de Educación Superior Abierta y a Distancia (ESAD), antecedente de la Universidad Abierta y a Distancia de México (UnADM); es decir, solo se apoyaba a estudiantes inscritos en esta universidad de modalidad no-presencial.

Con los datos expuestos, presentamos el contexto general de la inclusión educativa, desde su ámbito internacional y sus particularidades a nivel nacional. Sobre este escenario se desarrolla la presente investigación.

\section{REFERENTE TEÓRICO}

En relación con los modelos teóricos empleados sobre el tema de la discapacidad se encontraron diversas perspectivas, que fueron clasificadas de la siguiente manera:

a) La biomédica, que concibe la discapacidad como una enfermedad y describe sus deficiencias en términos morfológicos o funcionales (en ocasiones trata de curar/minimizar dicha condición).

b) La discapacidad como segregación, que concibe a esta condición como un lastre social.

c) La socioantropológica, que da relevancia a la existencia de una cultura alrededor de la discapacidad y que genera procesos, prácticas y conocimientos propios.

d) Del modelo social, que presenta la discapacidad como oprimida en la estructura social, lo que origina exclusión y marginación en este sector de la población.

e) Del modelo universal, que posee la perspectiva más reciente en la que la discapacidad es vista como parte de la condición humana; es decir, los seres humanos son seres vulnerables y expuestos a diversas circunstancias, por lo que en algunos momentos del ciclo vital requerirán apoyo del medio para su desenvolvimiento pleno (Cobos y Moreno, 2014; Moreno, 2011; Dubet, 2011). En esta visión moderna, Victoria (2013) propone encaminar los esfuerzos para que la población con discapacidad se encuentre en condiciones de igualdad y tenga acceso al pleno ejercicio de sus derechos y oportunidades, además de ofrecerles las condiciones para desarrollarse libremente, con dignidad y acorde a sus planes de vida.

Si bien en el presente artículo se parte de la idea de que el modelo universal es adecuado por su aspecto aspiracional -ya que permite trazar el rumbo que hemos de emprender-, también debe considerarse que ciertas vicisitudes pueden impedir la concreción de una inclusión adecuada. El modelo social nos permite observar estos aspectos negativos que redundan en la segregación o la exclusión. Cabe aclarar que esta perspectiva tiene diversos enfoques, sin embargo, todos coinciden en que la 
inclusión le compete principalmente a la sociedad y los gobiernos, y no solo a los sujetos en lo individual; en este sentido, es indispensable entender el contexto que los rodea y cómo este los acoge o los rechaza (Victoria, 2013).

En relación con los conceptos que anteceden la inclusión educativa, destaca la educación especial, la cual contribuyó al reconocimiento de las personas con discapacidad y en la distinción para ofrecerles una educación especializada; se podría decir que se reconoce a la educación especial como un proceso de intervención educativa que impulsó posteriormente el modelo integrador.

En este sentido surgió la educación integrada, con el propósito de preparar a los estudiantes con discapacidad para la vida y profesión futuras en un contexto más representativo de la sociedad. Es importante mencionar que el paso del concepto integración al de inclusión no solo fue una cuestión semántica, ya que esta última admite la diversidad en los procesos educativos y es un factor favorecedor del desarrollo humano (Parra, 2011).

En este sentido, la educación inclusiva no se limita a facilitar el acceso a las instituciones educativas para los alumnos con algún tipo de discapacidad, pues garantizar la accesibilidad únicamente sería reproducir un sistema de segregación inaceptable que terminaría en la expulsión de todo ese alumnado hacia un sistema ordinario que no ha cambiado. De acuerdo con Armstrong, Armstrong y Barton (2000), el sistema escolar que conocemos, en términos de factores físicos, aspectos curriculares, expectativas, estilos del profesorado y roles directivos, tendrá que cambiar.

La educación inclusiva no se reduce a la conciencia sobre el derecho a la educación de las personas con alguna discapacidad, con aptitudes sobresalientes o que pertenecen a cualquier otro grupo vulnerable; su esencia es la igualdad de oportunidades para todos mediante la eliminación de las barreras que existen, sean estas arquitectónicas, sociales o culturales, que limitan su participación o aprendizaje en el sistema educativo (Gallegos y López, 2018). Esta conceptualización nos permite entender el entramado de retos y desafíos que plantea favorecer una educación incluyente para las naciones y sus instituciones educativas.

La educación y las políticas públicas son temas inseparables a partir del supuesto de que no hay una agenda pública que no resalte el papel de la educación como instrumento de desarrollo económico y social o como encauzamiento de progreso (Carro y Lima, 2015). El campo de las políticas públicas estudia diversos aspectos relacionados con: a) los procesos de definición de los objetivos públicos del Estado; b) el desarrollo de organizaciones y programas dirigidos a alcanzar dichos objetivos, y c) el impacto de estos programas (Méndez, 2010). Por lo anterior, se vuelve indispensable entender los objetivos que trazan los Estados en sus planes nacionales y locales, específicamente respecto al tema de la inclusión educativa, pues estos mecanismos 
son los que impulsan acciones concretas a su favor, aunado a las acciones que las instituciones educativas puedan hacer, o no, al margen de las políticas.

Es indispensable entender que las instituciones son la célula donde se concretiza el plano normativo, las políticas públicas y la agenda gubernamental. En este contexto, la noción de institución educativa inclusiva se vuelve relevante porque debe ser capaz de responder, desde su organización, a las necesidades específicas de los alumnos y proporcionarles las herramientas que estos requieran, independientemente de sus características culturales, raciales, lingüísticas, de salud, entre otras (Escandón, 2010).

\section{Metodología}

\section{Enfoque}

El enfoque de la presente investigación es mixto, con un acercamiento exploratorio. Por una parte, se consideran aspectos de tipo cuantitativo relacionados con la construcción de un indicador que sirvió como parámetro de medida para el análisis del fenómeno de la inclusión educativa en instituciones educativas en México; por otra, se analizaron los datos obtenidos desde un aspecto cualitativo, descriptivo y teórico, fundamentado en la bibliografía revisada.

\section{Unidades de análisis}

Para cumplir con el objetivo de estudio planteado se diseñó un indicador que permitió medir el desempeño de las instituciones educativas en México en términos de inclusión educativa en el nivel superior, específicamente en la cobertura educativa para personas con discapacidad.

De acuerdo con Oakes (1986, citado en Tiana, 2011), los indicadores son los medios de evaluación empleados con mayor frecuencia en los temas de políticas públicas debido a que "permiten revelar algo sobre su funcionamiento o su estado de salud" (p. 21). Si bien la definición de Oakes es vigente, es importante señalar que los indicadores son herramientas cuantitativas que proporcionan información relevante acerca de aspectos significativos del ámbito educativo.

En este sentido, la evaluación permite, a través de los indicadores, emitir un juicio de valor, pues conduce a una opinión fundada de que algo es de cierto modo o se comporta de una forma determinada debido a su carácter comparativo, de acuerdo con el cual algo es mejor o peor a partir de considerar diversos criterios y normas dentro de un universo posible; por lo tanto, la evaluación redunda en una calificación o clasificación con base en el cumplimiento de estos principios (House, 1994). 


\section{Técnicas de recolección}

Para la recolección de datos se empleó la numeraria estadística de la Asociación $\mathrm{Na}$ cional de Universidades e Instituciones de Educación Superior (ANUIES) del ciclo escolar 2017. También se utilizó la estadística de población y vivienda del año $2014^{4}$ del Instituto Nacional de Estadística y Geografía ${ }^{5}$ (INEGI, 2014). Mediante el cruce de ambas bases de datos se construyó el indicador que mide la cobertura educativa de cada entidad federativa de México.

\section{Procesamiento de análisis}

A partir de los datos mencionados se obtuvo el porcentaje que cada estado presenta respecto a la población en edad escolar, en contraste con el número de alumnos inscritos en las instituciones. La construcción del indicador de inclusión se realizó a partir de la absorción de la matrícula con discapacidad, es decir, la población con discapacidad en edad escolar por entidad federativa sobre la matrícula con discapacidad inscrita en instituciones de educación superior.

\section{Construcción del indicador: $\mathrm{N}=(a / b) 100$}

donde $a$ representa la matrícula de estudiantes con discapacidad en instituciones de educación superior; $b$ es la población por entidad federativa de 15 a 24 años con discapacidad; $N$ resulta de la operación algebraica y representa el indicador de inclusión.

Con los resultados de la información estadística se construyeron tres categorías de desempeño para el indicador: inclusión baja, media y alta. La clasificación se determinó de acuerdo con el límite superior de los datos 20.82 y el límite inferior 0.47 (ver tabla 1), distribuido entre las tres categorías, lo que resultó en la siguiente clasificación:

- De 0.47 a 7.26, inclusión baja (rojo).

- De 7.27 a 14.05, inclusión media (azul).

- De 14.06 a 20.82, inclusión alta (verde).

La hipótesis construida para el presente artículo es: el marco normativo, las políticas gubernamentales y las acciones de las IES han impulsado la inclusión educativa en un escenario un tanto desorganizado, lo que ocasiona una cobertura desigual, con un crecimiento de la matrícula incluyente diferente en cada región.

4 Esta encuesta es la más reciente por parte del INEGI, ya que de acuerdo con la metodología de levantamiento se realiza de manera quinquenal.

5 Organismo público autónomo responsable de normar y coordinar el Sistema Nacional de Información Estadística y Geográfica, así como de captar y difundir información de México en cuanto al territorio, los recursos, la población y la economía, que permita dar a conocer las características de nuestro país y ayudar a la toma de decisiones (ver INEGI, s.f.). 
Tabla 1. Tabulador del indicador de cobertura para personas con discapacidad por entidad federativa.

\begin{tabular}{|c|c|c|c|c|}
\hline Núm. & Estados & $\begin{array}{l}\text { Población por entidad } \\
\text { federativa de } 15 \text { a } 24 \text { años } \\
\text { con discapacidad }(b)\end{array}$ & $\begin{array}{c}\text { Matrícula de estudiantes } \\
\text { con discapacidad en } \\
\text { IES }(a)\end{array}$ & $\begin{array}{l}\text { Indicador de } \\
\text { inclusión }(N) \\
(\%)\end{array}$ \\
\hline 1 & Aguascalientes & 4277 & 82 & 1.92 \\
\hline 2 & Baja California & 13052 & 1281 & 9.81 \\
\hline 3 & Baja California Sur & 3278 & 661 & 20.16 \\
\hline 4 & Campeche & 3425 & 175 & 5.11 \\
\hline 5 & Coahuila & 8654 & 911 & 10.53 \\
\hline 6 & Colima & 3410 & 270 & 7.92 \\
\hline 7 & Chiapas & 14005 & 312 & 2.23 \\
\hline 8 & Chihuahua & 15826 & 75 & 0.47 \\
\hline 9 & Ciudad de México & 27135 & 3405 & 12.55 \\
\hline 10 & Durango & 8568 & 182 & 2.12 \\
\hline 11 & Guanajuato & 21369 & 651 & 3.05 \\
\hline 12 & Guerrero & 14080 & 137 & 0.97 \\
\hline 13 & Hidalgo & 10360 & 2157 & 20.82 \\
\hline 14 & Jalisco & 38013 & 999 & 2.63 \\
\hline 15 & Estado de México & 67542 & 2925 & 4.33 \\
\hline 16 & Michoacán & 20529 & 278 & 1.35 \\
\hline 17 & Morelos & 6900 & 124 & 1.80 \\
\hline 18 & Nayarit & 6426 & 231 & 3.59 \\
\hline 19 & Nuevo León & 17966 & 1172 & 6.52 \\
\hline 20 & Oaxaca & 16965 & 2208 & 13.02 \\
\hline 21 & Puebla & 20961 & 1026 & 4.89 \\
\hline 22 & Querétaro & 7752 & 1250 & 16.12 \\
\hline 23 & Quintana Roo & 5304 & 247 & 4.66 \\
\hline 24 & San Luis Potosí & 11749 & 606 & 5.16 \\
\hline 25 & Sinaloa & 12607 & 425 & 3.37 \\
\hline 26 & Sonora & 10392 & 991 & 9.54 \\
\hline 27 & Tabasco & 9096 & 580 & 6.38 \\
\hline 28 & Tamaulipas & 11673 & 768 & 6.58 \\
\hline 29 & Tlaxcala & 4401 & 254 & 5.77 \\
\hline 30 & Veracruz & 34798 & 522 & 1.50 \\
\hline 31 & Yucatán & 8911 & 319 & 3.58 \\
\hline \multirow[t]{2}{*}{32} & Zacatecas & 7541 & 702 & 9.31 \\
\hline & Total & 466964 & 25926 & 5.55 \\
\hline
\end{tabular}

Fuente: Elaboración propia con datos del INEGI (2014) y la ANUIES (s.f.). 


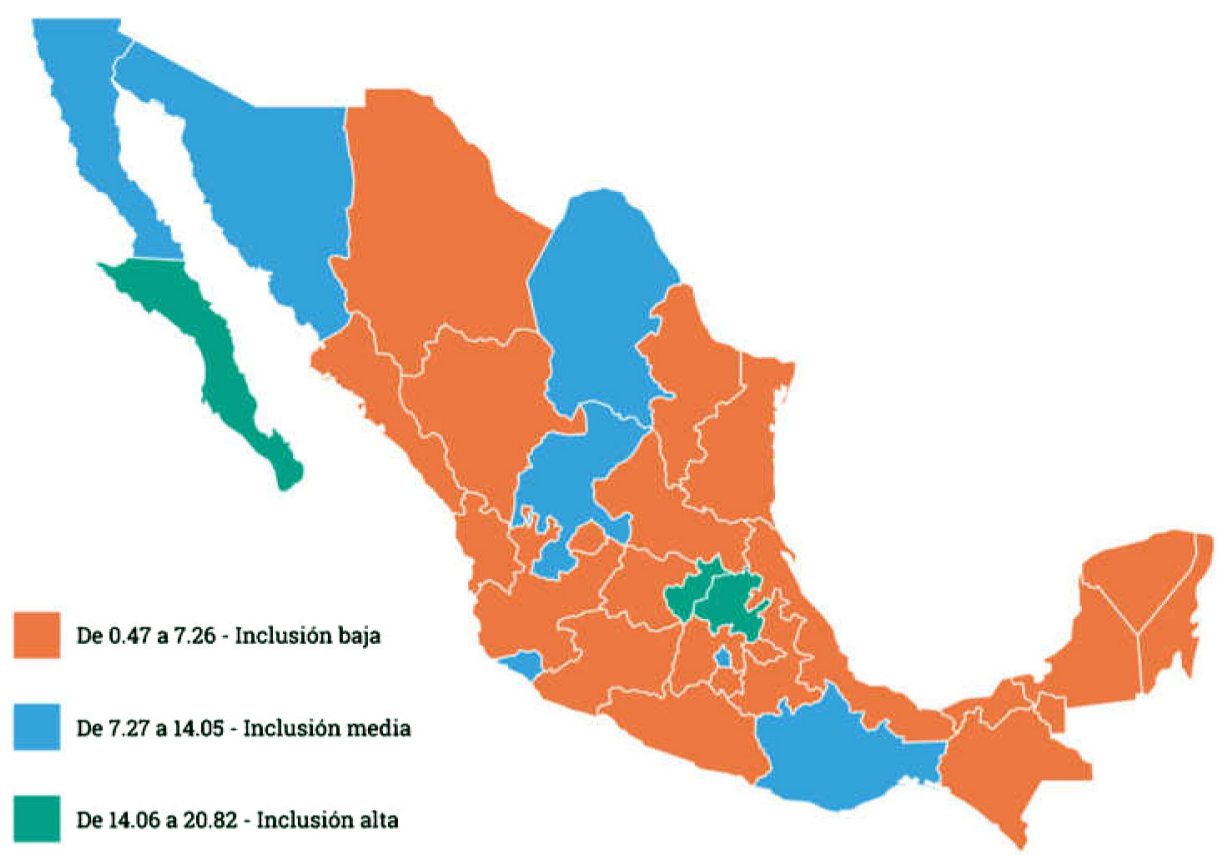

Figura 2. México: indicador de inclusión educativa por entidad federativa. Fuente: Elaboración propia con datos del INEGI (2014) y la ANUIES (s.f.).

\section{Resultados}

Los estados con mayor inclusión en la República mexicana son: Hidalgo, Baja California Sur y Querétaro. Los estados con una inclusión media son: Oaxaca, Ciudad de México, Coahuila, Baja California, Sonora y Zacatecas. El resto tuvo un desempeño deficiente respecto del indicador (inclusión baja). Este escenario desesperanzador hace mella también en algunas de las principales urbes de la nación (Estado de México, Nuevo León y Jalisco), de las cuales se esperaría mayor impacto en el tema de inclusión. Sin embargo, la metrópoli más importante de la nación, Ciudad de México, es la que concentra el mayor número de matrícula con algún tipo de discapacidad, aunque ese número representa poco respecto de la población total con discapacidad en edad escolar (de 15 a 24 años) en esa entidad; esta región tuvo un índice catalogado como "inclusión media" (ver figura 2 y tabla 1 ).

En México existe un promedio nacional de cobertura de inclusión educativa en el nivel superior de solo 5.55\%; es decir, de los 466,964 jóvenes con algún tipo de discapacidad que residen en nuestro país, solo 25,926 se encuentran inscritos en alguna institución educativa. Cabe señalar que la cobertura educativa en este nivel, a escala nacional, es de $38.47 \%$ entre la población sin discapacidad. Este panorama indica que la inclusión educativa no parece tener relación con las grandes metrópolis que tienden a poseer las más grandes instituciones de educación superior, con presupuestos abultados y con mayor cobertura de matrícula no inclusiva. 
Respecto a la comparación del sector público y privado, la cobertura educativa se muestra polarizada, como se evidencia en la tabla 2.

Tabla 2. Distribución de la matrícula de inclusión por tipo de financiamiento.

\begin{tabular}{ccc} 
Tipo & Matrícula & Porcentaje \\
\hline Pública & 23,585 & $91 \%$ \\
\hline Privada & 2,341 & $9 \%$ \\
\hline Total & 25,926 & $100 \%$ \\
\hline
\end{tabular}

Fuente: Elaboración propia.

\section{Discusión}

Estos datos evidencian la importancia de la educación pública en la agenda de la inclusión educativa en México, pues en este sector se encuentra el 91\% del total de la matrícula con discapacidad. En virtud de esto, se vuelven relevantes las acciones que realiza el ministerio de educación en México (Secretaría de Educación Pública), así como los recursos humanos y económicos destinados a este nivel educativo; es decir, las políticas como instrumentos, normas y recursos son el motor que propiciaría el cambio hacia la reducción de las brechas de exclusión.

En contraste con lo establecido en los programas de financiamiento que apoyan la inclusión de personas con discapacidad en la educación superior, la mayor cantidad de esta matrícula se encuentra en la modalidad escolarizada, con 22,774 estudiantes, lo cual representa $88 \%$ de esta población estudiantil, mientras que la modalidad no escolarizada registra tan solo $12 \%$, lo que representa un total de 3,152 estudiantes, como puede observarse en la tabla 3.

Tabla 3. Población escolar con discapacidad por tipo de modalidad.

\begin{tabular}{ccc} 
Tipo & Matrícula & Porcentaje \\
\hline Escolarizada & 22,774 & $88 \%$ \\
\hline No escolarizada & 3,152 & $12 \%$ \\
\hline Total & 25,926 & $100 \%$ \\
\hline
\end{tabular}

Fuente: Elaboración propia.

La mayoría de los estudiantes con discapacidad eligen las instituciones tradicionales, es decir, la modalidad presencial, por lo que este tipo de universidades debe contar con los requerimientos de accesibilidad universal: rampas, elevadores, señaléticas braille y auditivas, losetas podotáctiles, puertas de apertura automáticas, así como adaptaciones de espacios físicos como aulas, baños, bibliotecas, etcétera.

Otros aspectos importantes que deben considerarse para la eliminación de las barreras educativas son: la capacitación docente, el diseño de modelos educativos y 
pedagógicos incluyentes. Además, es necesario promover la eliminación de las barreras sociales mediante la capacitación del personal universitario y la concientización de la comunidad estudiantil, en general. Los cumplimientos de todas estas medidas contribuirían a una inclusión en el sentido estricto de la definición.

Los estudiantes con discapacidad acuden mayoritariamente a las instituciones presenciales, justo como sería lo idóneo para cualquier joven universitario, vivir la universidad en todos sus ámbitos: escolar, social y cultural. Los hechos eran diferentes a las intenciones de los programas gubernamentales en el periodo 2009-2013, ya que los apoyos de becas para personas con discapacidad fueron para aquellos estudiantes que acudían a la modalidad abierta y a distancia del Programa Educación para Personas con Discapacidad.

En menor medida, existe un sector de la población con discapacidad que opta por otras modalidades no convencionales (12\%). En este sentido, la educación a distancia puede convertirse en una opción complementaria para aumentar la cobertura educativa, no solo en la matrícula tradicional, sino también para quienes tienen algún tipo de discapacidad. Es importante señalar que las personas en esta condición pueden estar expuestas a diversas dificultades para asistir a las universidades de manera presencial, obstáculos que no necesariamente están ligados a su discapacidad, sino a las condiciones o los ritmos de su vida cotidiana como su empleo, sus responsabilidades familiares, entre otras.

Si bien la población con discapacidad se ha incorporado de manera gradual en la educación no-convencional, es necesario que las instituciones que ofertan estas modalidades puedan integrar a sus alumnos de manera inclusiva, esto quiere decir que requerirán enfocar esfuerzos de accesibilidad a sus plataformas educativas, en el caso de la modalidad virtual, así como de reducir brechas educativas y sociales en sus ambientes de aprendizaje.

A pesar de que las propuestas en las modalidades no-convencionales de la educación, como la semipresencial, abierta, virtual o a distancia, no han sido consideradas como una solución para incrementar el porcentaje de la inclusión en la educación, esto es una tarea pendiente. Deben incorporarse nuevos programas de estudio que contemplen las necesidades de la población estudiantil, en particular, las que corresponden a grupos minoritarios, como quienes padecen alguna discapacidad, ya que estos sectores requieren de medidas apropiadas a su condición.

\section{ConClusión}

En resumen, el panorama general de la inclusión educativa en el nivel superior en México es incipiente; aún falta mucho por hacer y lograr. Los pocos esfuerzos que han rendido frutos más evidentes se han logrado de manera dispersa en el territorio nacional. Las principales urbes (Ciudad de México, Monterrey y Jalisco) aún presentan 
una baja cobertura de inclusión educativa. Por ende, el primer paso será incrementar esta cobertura: una meta idónea sería llegar a la mitad del indicador de cobertura en educación superior, que es de $13.15 \%$, en virtud de que la cobertura en este nivel durante el ciclo 2014-2015 fue de 26.3\% de matrícula no inclusiva.

México cuenta con un promedio nacional de cobertura de inclusión educativa en educación superior de 5.55\%, una brecha importante sobre la cual trabajar, ya que la diferencia es de $7.60 \%$. Por lo tanto, es indispensable reducir este rezago, pues actualmente solo poco más del $5 \%$ de las personas con discapacidad y con una edad de entre 18 y 24 años se encuentran matriculadas en alguna institución de educación superior en el país. Esto implica que la educación básica también tendrá que aumentar su cobertura de inclusión, pues estas instituciones formarán a esta población dispuesta a continuar hacia la universidad.

Si bien el primer paso para la inclusión educativa es garantizar el acceso a la educación, ya sea para instituciones públicas o privadas que ofrecen educación escolarizada u otras modalidades no-convencionales, uno de los desafíos presentes para garantizar una plena integración educativa es consolidar la accesibilidad universal en sus instalaciones físicas. Sin embargo, el reto a futuro para las instituciones será ofrecer las condiciones que permitan la permanencia de los estudiantes con discapacidad y, a su vez, enfocar estrategias para redundar en la culminación de sus estudios universitarios, retos que requerirán principalmente de reducir las brechas educativas en las IES y las barreras sociales.

Las estrategias de inclusión educativa que llevan a cabo las instituciones de educación superior en México son bastante diversas y, como lo demuestra Pérez-Castro (2016), muy pocas universidades públicas y privadas tienen información más o menos sistematizada. Por lo tanto, será indispensable generar un sistema de información e indicadores de manera centralizada, que permita aprovechar los censos escolares de la SEP para recabar información concerniente al estado que guarda la inclusión educativa en las IES; ${ }^{6}$ es decir, es prioritario generar un diagnóstico integral.

Además, será fundamental incrementar los recursos económicos y diversificar los programas operativos nacionales que apoyen la inclusión educativa; esto en virtud de que los implementados actualmente son insuficientes por su escaso financiamiento y el impacto de los mismos solo ha bordeado el ámbito de la accesibilidad universal en las IES; en tanto que otros programas han dado prioridad a sectores vulnerables como personas en situación de pobreza y pertenecientes a grupos étnicos.

Si bien las IES han realizado acciones para favorecer la inclusión educativa para personas con discapacidad, parece que esta labor es atomizada y aislada, es decir,

6 La encuesta anual de Inicio de curso 2018-2019 que implementa la SEP (cuestionario 911) ha incorporado recientemente nuevos ítems en su estructura, agregando el módulo "Infraestructura de educación especial". 
no existe una clara coordinación entre el Estado y las IES, pues las acciones entre ambas partes no reflejan un impacto sobre la demanda educativa de este sector de la población. Podríamos inferir que las que obtuvieron mejores resultados fueron instituciones con agendas inclusivas muy claras, lo que les ha permitido optimizar sus propios recursos y los apoyos gubernamentales en favor de la inclusión. Esto nos lleva a afirmar que la inclusión educativa no solo es un asunto de buenas voluntades, sino de políticas públicas, programas, recursos y acciones concretas, que son evaluadas constantemente para entender su impacto, alcances y retos futuros.

\section{REFERENCIAS}

Armstrong, F., Armstrong, D., y Barton, L. (eds.) (2000). Inclusive education: Policy, contexts and comparative perspectives. Londres: David Fulton Publishers.

ANUIES [Asociación Nacional de Universidades e Instituciones de Educación Superior] (s.f.). Asociación Nacional de Universidades e Instituciones de Educación Superior. Recuperado de: http://www.anuies.mx/.

Benavides, M., Maz, A., Castro, E., y Blanco, R. (2004). La educación de niños con talento en Iberoamérica. Santiago, Chile: UNESCO.

Carro, A., y Lima, J. (2015). Políticas públicas para la inclusión educativa, el caso del Programa de Estímulos a la Educación Superior en Terrenate, Tlaxcala. Revista Espacios Públicos, 18(44), 125-144. México: Universidad Autónoma del Estado de México.

Cobos, A., y Moreno, M. (2014). Educación superior y discapacidad: análisis desde la experiencia de algunas universidades colombianas. Revista Española de Discapacidad, 2(2): 83-101.

Congreso de la Unión de los Estados Unidos Mexicanos (2003). Ley Federal para Preveniry Eliminar la Discriminación. Ciudad de México: Congreso de la Unión.

Congreso de la Unión de los Estados Unidos Mexicanos. (2011). Ley General para las Personas con Discapacidad. Ciudad de México: Congreso de la Unión.

Cruz Bilbado, M. (2010). Recursos que favorecen la inclusión de estudiantes con discapacidad en la educación superior según los docentes universitarios. International Journal of Developmental and Educational Psychology, 4(1), 777-787.

Cruz, R. (2016). Discapacidad y educación superior: ¿Una cuestión de derechos o buenas voluntades? CPU-E Revista de Investigación Educativa, 1-23.
Definición.mx (2014, mar. 6). Inclusión. Recuperado de: https://definicion.mx/inclusion/(consulta: 10 ago. 2018).

Dubet, F. (2011). Repensar la justicia social. Contra el mito de la igualdad de oportunidades. México: Siglo XXI.

Escandón, M. C. (2010). Guía para facilitar la inclusión de alumnos y alumnas con discapacidad en escuelas que participan en el Programa Escuelas de Calidad. México: Secretaría de Educación Pública.

Gallegos, T., y López, F. (2018). Inclusión y equidad en el nuevo modelo educativo. Revista de Administración Pública, 53(1).

Gobierno de México (s.f.). Programa para la Inclusión y la Equidad Educativa. Recuperado de: http://www. dgesu.ses.sep.gob.mx/inclusion_y_equidad.htm.

Gobierno de México (s.f.). Prospera Programa de Inclusión Social. Gobierno de México. Recuperado de: https://www.gob.mx/bienestar/ acciones-y-programas/prospera-programa-deinclusion-social-15908\#: : :text $=$ noviembre $\% 20$ de $\% 202015-$,E $1 \% 20$ Programa $\% 20$ de $\% 20$ Inclusi $\% \mathrm{C} 3 \% \mathrm{~B} 3 \mathrm{n} \% 20$ Social $\% 20$ PROSPERA $\% 20$ articula $\% 20 y \% 20$ coordina $\% 201 a, y \% 20$ salud $\% 2$ C $\% 20$ dirigida $\% 20 \mathrm{a} \% 201 \mathrm{la}$.

House, E. (1994). Evaluación ética y poder. España: Morata.

INEGI [Instituto Nacional de Estadística y Geografía] (2014). Censo de población y vivienda. Recuperado de: https://www.inegi.org.mx/.

INEGI (s.f.). Instituto Nacional de Estadística y Geografía. Recuperado de: https://www.inegi.org.mx/.

Méndez, J. (2010). La política pública como variable dependiente: hacia un análisis más integral de las 
políticas públicas. En Política pública. México: Siglo XXI.

Moreno, M. (2011). Infancia, políticas y discapacidad. Bogotá: Unibiblos.

Parra, C. (2011). Educación inclusiva: un modelo de diversidad humano. Educación y Desarrollo Social, (1), 139-150.

Pérez-Castro, J. (2016). La inclusión de las personas con discapacidad en la educación superior en México. Sinéctica, Revista Electrónica de Educación, 1-15.

PND (2013). Plan Nacional de Desarrollo. México.

SHCP [Secretaría de Hacienda y Crédito Público] (s.f.). Transparencia presupuestaria. Observatorio del gasto. Recuperado de: https://www.transparenciapresupuestaria. gob.mx/.

Secretaría General de la OEA (2001). Convención Interamericana sobre la Eliminación de todas las Formas de Discriminación contra las Personas con Discapacidad. OEA. Recuperado de: https://www.oas.org/juridico/spanish/firmas/a-65.html.

Tapia Berrios, C., y Manoslava Mena, S. (2012). Inclusión de estudiantes con discapacidad en la educación superior. REXE Revista de Estudios y Experiencias en Educación, 11(22), 13-34.

Tiana Ferrer, A. (2011). Los sistemas de indicadores: una radiografía de la educación. En M. Kisilevsky y E. Roca (coords.), Indicadores, metas y politicas educativas. Madrid, España: Organización de Estados
Iberoamericanos para la Educación, la Ciencia y la Cultura (OEI).

Toscano de la Torre, B. A., Ponce Gallegos, J. C., Cruz Juárez, A. D., Zapién de la Torre, A., Contreras Vega, G., y Pérez Arriaga, J. C. (2017). Análisis de la inclusión en la educación superior en México. Una propuesta para los organismos acreditadores. Tecnología Educativa Revista CONAIC, 4(2).

UNESCO [Organización de las Naciones Unidas para la Educación, la Ciencia y la Cultura] (1994). Declaración de Salamanca y Marco de Acción para las Necesidades Educativas Especiales. Salamanca: UNESCO.

UNESCO (2008). Convención sobre los Derechos de las Personas con Discapacidad. Nueva York: UNESCO.

Vadillo, R., y Casillas, M. (2017). Las instituciones de educación superior y los estudiantes con discapacidad en México. Revista de la Educación Superior, 46(181), 37-53.

Victoria, J. (2013) El modelo social de la discapacidad: una cuestión de derechos humanos. En Boletín Mexicano de Derecho Comparado, 46(138, nueva serie), 1093-1109.

Villafañe Hormazábal, G. P., Corrales Huenul, A. A., y Soto Hernández, V. J. (2014). Estudiantes con discapacidad en una univerdiad chilena: desafíos de la inclusión. Revista Complutense de Educación, 27(1), 353-372.

Zárate-Rueda, R., Díaz-Orozco, S. P., y Ortiz-Guzmán, L. (2017). Educación superior inclusiva: un reto para las prácticas pedagógicas. Revista Electrónica Educare. doi: http://dx.doi.org/10.15359/ree.21-3.15.

Cómo citar este artículo:

González García, J. A., Zúñiga Llamas, A., y Arce Casas, P. O. (2021). Un panorama sobre la cobertura educativa a nivel superior en México para personas con discapacidad. IE Revista de Investigación Educativa de la REDIECH, 12, e1171. doi: 10.33010/ie_rie_rediech.v12i0.1171. 